\title{
Preserving Polar Modulated Class-E Power Amplifier Linearity under Load Mismatch
}

\author{
Awani Khodkumbhe ${ }^{\# 1}$, Maikel Huiskamp $\$$, Ali Ghahremani ${ }^{\$}$, Bram Nauta ${ }^{\$}$, Anne-Johan Annema ${ }^{\$ 2}$ \\ \#BITS Pilani, India \\ ${ }^{\$}$ University of Twente, The Netherlands \\ 1awani678@gmail.com,2a.j.annema@utwente.nl
}

\begin{abstract}
Power amplifiers (PAs) need digital predistortion (DPD) linearization to handle high-order complex modulation schemes in next-generation communication systems. While load variation is inevitable, DPD is generally designed considering only the nominal load impedance for PAs. This paper presents a polar class-E PA with an on-chip waveform characterizer enabling adaptive digital predistortion (ADPD) to preserve the linearity of the PA under load mismatch. The presented ADPD corrects both $\mathrm{AM} / \mathrm{AM}$ and $\mathrm{AM} / \mathrm{PM}$ distortions, which are prominent in the demonstrated PA, while simultaneously correcting for slow memory effects without the need for complex memory DPD algorithms. Load-pull measurements demonstrate that target error vector magnitude (EVM) and adjacent channel power ratio (ACPR) can be maintained in a significantly larger area on the Smith chart going from $50 \Omega$ optimized static DPD to our ADPD for a $2 \mathrm{GHz} 1024 \mathrm{QAM}$ signal with $1 \mathrm{MSym} / \mathrm{s}$ symbol rate.

Keywords-CMOS integrated circuits, power amplifiers, linearity, distortion, predistortion, impedance, antennas, adaptive control, quadrature amplitude modulation.
\end{abstract}

\section{INTRODUCTION}

Modern wireless communication devices use high-order complex modulation schemes like 1024 QAM. These signals have a high peak to average power ratio (PAPR) and impose stringent linearity requirements on the power amplifiers (PAs). Given the large share of power consumed by the PAs in a communication system, highly efficient switched-mode PAs are attractive. However, due to inherent efficiency-linearity trade-offs [1], digital predistortion (DPD) becomes necessary for these PAs to meet linearity requirements [2], [3] of increasing order complex modulation schemes.

The antenna impedance depends on the electromagnetic environment of the antenna and therefore vary over time [4]. In switched-mode PAs, load mismatch from the nominal $50 \Omega$ load detunes the resonant network. For class-E PAs, this degrades the output power, efficiency, linearity and also jeopardizes the reliability [5]. For a polar class-E PA, this translates to variation in $\mathrm{AM} / \mathrm{AM}$ and $\mathrm{AM} / \mathrm{PM}$ characteristics, which renders $50 \Omega$ optimized static DPD ineffective under load variation [6].

Previous works use various techniques to preserve PA linearity under load mismatch. These can be categorized into methods that keep the PA load impedance at its nominal value, and methods that deal with PA load variation. Implementations of the former methods are presented in [7], [8]. In [7], an adaptive tunable matching network (TMN) ensures the nominal load for the PA and thereby preserves linearity under antenna mismatch conditions. However, TMNs are

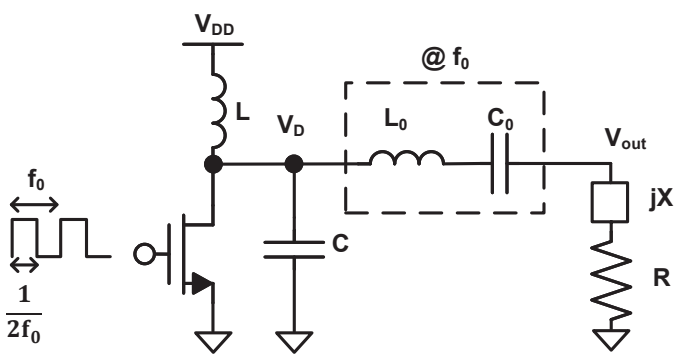

Fig. 1. Schematic representation of a single-ended class-E PA.

lossy, bulky and hence not suitable for easy integration in ever-miniaturizing CMOS integrated circuits. A solution with similar disadvantages is the addition of circulators or isolators with the PA. In [8], a phase detector at the collector of the PA combined with a controlled phase shifter at the output are used. The increase in size and high driving voltage prevents the above approach from being practically integrated into the latest CMOS technology. Implementation of the latter method is described in [9], where a peak detector at the collector of the PA combined with an adaptive feedback control loop to tune the input drive signal is used. However, such a system improves linearity at the cost of transmitted power because it reduces the input drive level. Another way to deal with load-dependent non-linearity is to implement DPD for different loads and select the suitable DPD setting using load impedance estimation. For class-E PAs, to the best of the authors' knowledge, there is no work focusing on integrated adaptive linearity improvement under severe load mismatch.

We present a proof-of-concept polar class-E PA with an integrated waveform characterizer, which allows the implementation of fully automated adaptive digital predistortion (ADPD) to preserve the linearity of a PA under load mismatch, aiming at 1024 QAM modulation.

In section II, the linearity of polar class-E PA under load mismatch is discussed. In section III, the principle of adaptive preservation of PA linearity with the integrated waveform characterizer and implementation of the system are discussed. The measurement results demonstrating the effectiveness of the system under load mismatch are presented in section IV. Conclusions are summarized in section V.

\section{LINEARITY OF A POLAR CLASS-E PA UNDER LOAD MISMATCH}

Fig. 1 depicts a single-ended (non-polar) class-E PA where the MOSFET operates as a switch driven by a $50 \%$ 


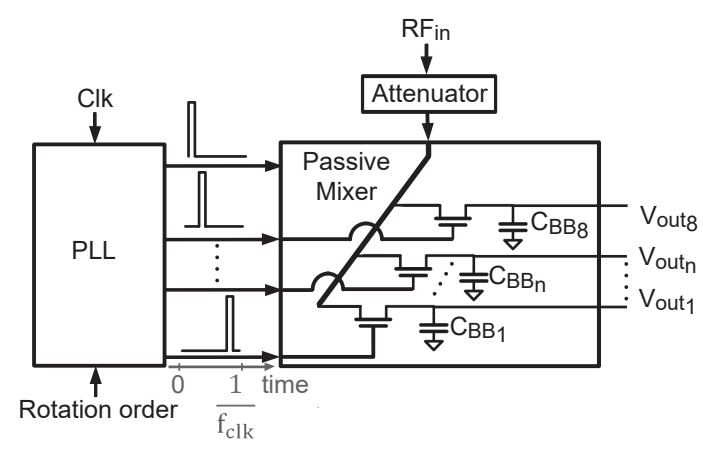

Fig. 2. Block diagram of RF waveform characterizer.

duty cycle square wave with a frequency $\mathrm{f}_{0}$. The LC tank shapes the switch's drain voltage waveform $\mathrm{V}_{\mathrm{D}}$, and the $\mathrm{L}_{0} \mathrm{C}_{0}$ resonant tank ensures that the output current is sinusoidal with frequency $\mathrm{f}_{0}$. A matching network (not in Fig. 1) transforms the antenna impedance to the PA's load impedance: $Z_{L}=$ $R+j X$. Voltage $V_{D}$ depends on both the real and imaginary parts of $Z_{L}$ [5]. This $Z_{L}$-dependent switch voltage translates to $Z_{\mathrm{L}}$-dependent magnitude and phase of the first harmonic of the output voltage signal of the class-E PA.

In communication systems, the PA must handle modulated signals. In this paper, we aim at 1024 QAM. To deal with complex modulated signals, the class-E PA can be used in outphasing or polar configuration; we use the latter.

In polar modulated class-E PA, distortions are due to $\mathrm{AM} / \mathrm{AM}$ and $\mathrm{AM} / \mathrm{PM}$ conversions. $\mathrm{AM} / \mathrm{AM}$ represents the non-linear relation between the modulated supply voltage $\mathrm{V}_{\mathrm{DD}}$ and the amplitude of the output RF signal $\mathrm{V}_{\text {out }}$. AM/PM is $\mathrm{V}_{\mathrm{DD}}$-dependent phase modulation of $\mathrm{V}_{\text {out }}$. Any change in $\mathrm{Z}_{\mathrm{L}}$ leads to a change in the class-E PA's switch voltage waveform and consequently leads to $\mathrm{Z}_{\mathrm{L}}$-dependent $\mathrm{AM} / \mathrm{AM}$ and $\mathrm{AM} / \mathrm{PM}$ characteristics. Other causes of $\mathrm{AM} / \mathrm{AM}$ and AM/PM conversions in a polar class-E PA include feedthrough of the drive voltage of the switch via its relatively large gate-drain capacitance, non-zero on-resistance of the switch, and drain-bulk junction capacitance of the switch [10]. Note that the feedthrough signal is only phase modulated and hence has a wide bandwidth. Therefore, it leads to broadening of the output spectrum, increasing the adjacent channel power ratio (ACPR). The cascode PA topology minimizes the effect of the feedthrough signal.

\section{DEMONSTRATION OF ADPD WITH POLAR CLASS-E PA}

We present a polar class-E PA with an on-chip RF waveform characterizer that enables ADPD to correct $\mathrm{AM} / \mathrm{AM}, \mathrm{AM} / \mathrm{PM}$ distortions, temperature changes and slow memory effects as the load changes.

\section{A. Waveform Characterizer}

The RF waveform characterizer is based on the work in [11]. Fig. 2 shows the block diagram of this waveform characterizer. It can measure up to three harmonics of an RF signal up to a fundamental frequency of $3 \mathrm{GHz}$. Hence, the waveform characterizer can be used for ADPD in PAs with

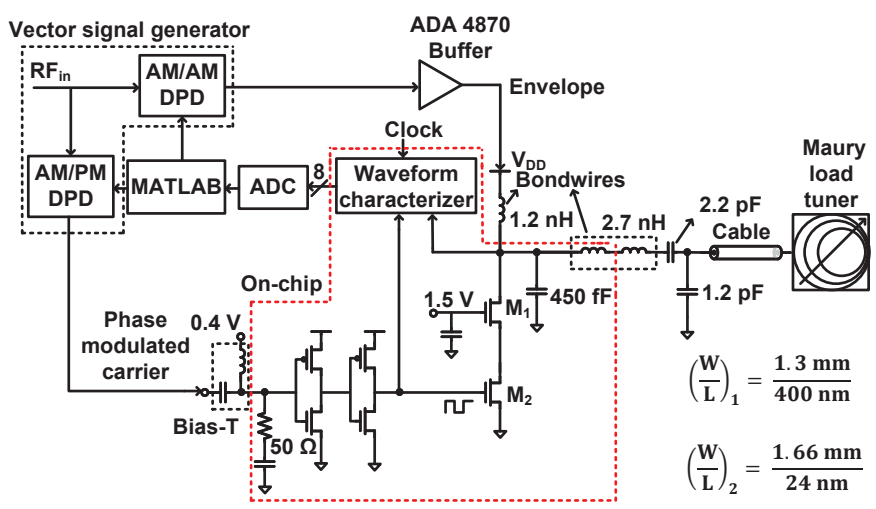

Fig. 3. Schematic of the implemented polar class-E PA with ADPD using the integrated waveform characterizer.

operating frequencies up to $3 \mathrm{GHz}$; we target $2.4 \mathrm{GHz}$ for demonstration.

The (internal) drain voltage signal in class-E PAs is harmonic rich. These harmonics are filtered by series $\mathrm{L}_{0} \mathrm{C}_{0}$ tank and external matching network to obtain fundamental harmonic at the output. Therefore, any feedback system operating on an internal PA signal must estimate the magnitude and phase of the first harmonic of the transmitted signal.

Using conventional loopback systems, harmonic rejection quadrature mixers are needed to obtain the magnitude and phase of first harmonic. A sufficiently accurate harmonic rejection quadrature mixer at RF frequency is difficult to achieve [2].

Our characterizer mixes the RF waveform using an N-path approach and produces 8 quasi-DC samples per period of the RF waveform. These 8 samples are input to ADCs, the output of which is processed in a computer running MATLAB. The waveform characterizer uses the (unmodulated) carrier frequency as the clock signal: an extra clock is not required and the phase information of the measured signal can be obtained. An 8-point discrete fourier transform (DFT) is performed in MATLAB to get the magnitude and phase information of the three harmonics of the sampled RF signal. In such systems, accuracy is generally limited by sample timing inaccuracies which in this case is mainly due to delay mismatch in a 4-stage differential inverter ring oscillator inside the phase-locked loop (PLL). To significantly enhance the accuracy of sampling, statistical properties of the delay mismatches are exploited to cancel the impact of delay spread [11]. The characterizer consumes $3 \mathrm{~mW}$ power under continuous operation at $2 \mathrm{GHz}$.

\section{B. Adaptively Preserving PA Linearity}

Fig. 3 presents the schematic of the polar modulated PA employing ADPD. For the ADPD, the input and output signal of the PA are required. The transmitted data is known in a transmitter while the waveform characterizer samples the modulated data at the drain of the switch. The DFT yields magnitude and phase information of the first 3 harmonics in the drain voltage signal; the fundamental harmonic is used for ADPD. Magnitude and phase data are compared with the corresponding transmitted magnitude and phase data derived 


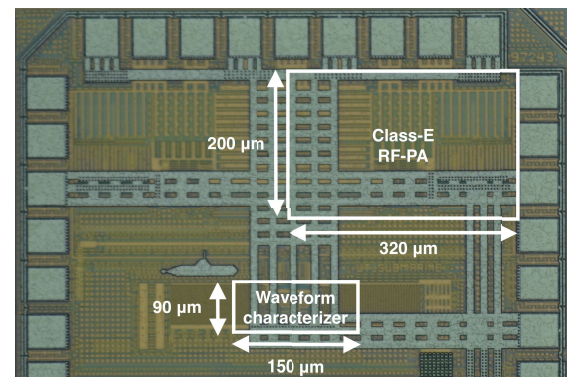

Fig. 4. Chip microphotograph.

from IQ data transmitted by the vector signal generator (VSG) to generate $\mathrm{AM} / \mathrm{AM}$ and $\mathrm{AM} / \mathrm{PM}$ correction signals. For flexibility, signal processing is done on a computer running MATLAB. The correction signals are then input to the lookup table (LUT) based DPD inside the VSG. The VSG outputs the predistorted envelope and phase signals for the polar class-E PA. The loop is executed periodically making the PA linearity robust against any relatively slow process that impacts linearity.

The time constant of the waveform characterizer is $\tau=16$ ns. For $40 \mathrm{~dB}$ accuracy, $5 \tau$ is required which makes the effective settling time of the characterizer $80 \mathrm{~ns}$. Hence the maximum symbol rate measured by the waveform characterizer is $12.5 \mathrm{Msym} / \mathrm{s}$. Transmitted and the sampled data are read from a mixed-signal scope in blocks. Reading one block takes $0.65 \mathrm{~s}$; reading data from 8 channels requires $5.2 \mathrm{~s}$. The bottleneck for effective sample rate in our demonstration is due to latency while reading data. Antenna environment changes on a time scale of ms [12]. Removing data latency bottleneck can reduce the response time of the complete system to order of ms making the system adapt to changes on a ms time scale such as temperature changes, slow memory effects [13] and antenna impedance variations.

\section{Implementation}

The chip includes a single-ended cascode class-E PA and the RF waveform characterizer fabricated in $22 \mathrm{~nm}$ FDSOI CMOS technology packaged in QFN 40; Fig. 4 shows the microphotograph. The PA occupies $0.064 \mathrm{~mm}^{2}$ area, and the waveform characterizer occupies $0.014 \mathrm{~mm}^{2}$ area. The PA is designed for zero voltage switching and zero slope switching conditions at frequency $\mathrm{f}_{0}=2.4 \mathrm{GHz}$. The peak-power frequency is shifted to $2 \mathrm{GHz}$ from the target $2.4 \mathrm{GHz}$, which may be due to the bondwires at the PA output. The component values for the designed PA are shown in Fig. 3. The $1.2 \mathrm{nH}$ inductor is implemented using bondwires; the parasitic capacitance of the MOSFET is included in the 450 $\mathrm{fF}$ capacitor. Two cascaded inverters are used as switch driver ensuring hard switching of the switch transistor. The input of the on-chip waveform characterizer is connected to the drain of switch and the outputs of the characterizer are fed to MATLAB through ADCs. The predistorted input signal is fed to the PA through an R\&S SMW200A VSG. An ADA 4870 IC implements the envelope tracking buffer for the demonstrator.

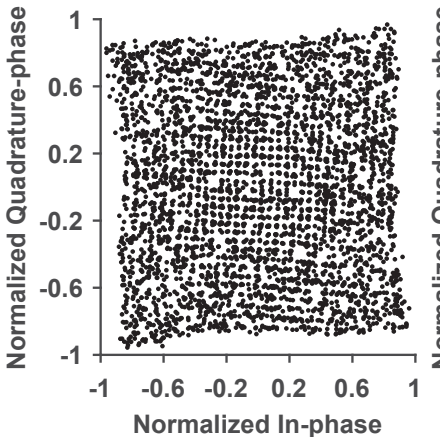

(a)

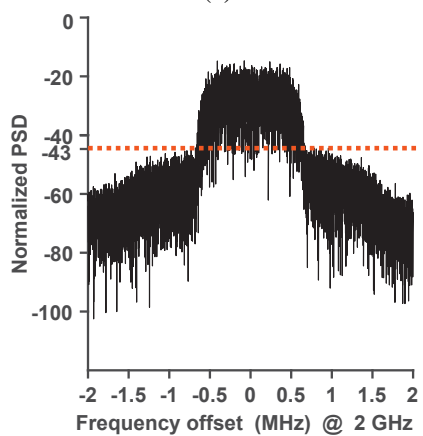

(c)

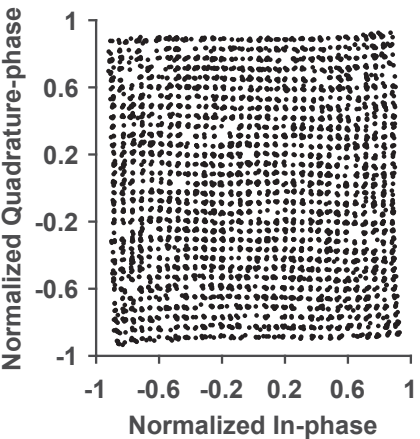

(b)

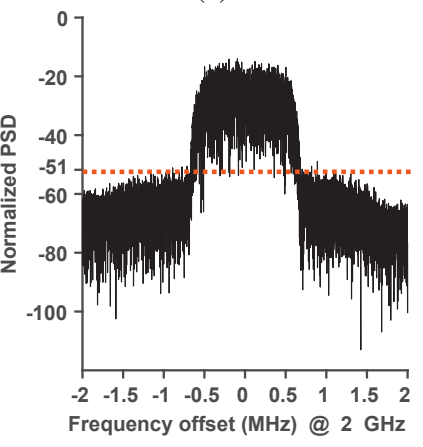

(d)
Fig. 5. Measured performance for a $1 \mathrm{MHz} 1024 \mathrm{QAM}$ signal at $2 \mathrm{GHz}$ at $50 \Omega$ load. Normalized symbol constellation (a) before DPD, (b) after DPD. Normalized output power spectral density (c) before DPD, (d) after DPD.

\section{MEASUREMENTS}

Single carrier modulated signal measurements are performed on the polar class-E PA to demonstrate linearity preservation using the presented ADPD under load mismatch. A 1024 QAM signal at $1 \mathrm{MSym} / \mathrm{s}$ symbol rate with pseudo-random bit sequence (PBRS) 23, pulse-shaped using root-raised cosine filter with a roll-off factor 0.35 and decomposed into the envelope and phase-modulated carrier at frequency $2 \mathrm{GHz}$ are generated using the VSG. 400 symbols are used to construct AM/AM and AM/PM correction signals. Load-pulling is performed with a Maury load-pull tuner for voltage standing wave ratio (VSWR) up to 9:1. The PA is operated at $15 \mathrm{~dB}$ back-off for the nominal load condition to avoid breakdown during load-pull [5] while no class-E tuning for reliability [14] is done in this work to focus only on the linearity. Fig. 5 (a), (b) show the normalized output symbol constellation and (c), (d) the normalized output power spectral density (PSD) before and after DPD, respectively at $50 \Omega$ load. Fig. 6 (a) and (b) show the Smith charts of EVM $(\%)$ and ACPR $(\mathrm{dB})$ of the polar class-E PA without any DPD. Fig. 7 (a) and (b) show Smith charts of EVM (\%) up to VSWR 9:1 with $50 \Omega$ optimized static DPD and with ADPD, respectively. The EVM is normalized to root mean squared (RMS) power. The EVM standard for 1024 QAM signal is considered $1.5 \%$. The load-pull Smith charts without DPD, with $50 \Omega$ optimized static DPD and with ADPD include a shaded area for which EVM $\leq 1.5 \%$. A significant increase in area under the shaded region can be observed from no 


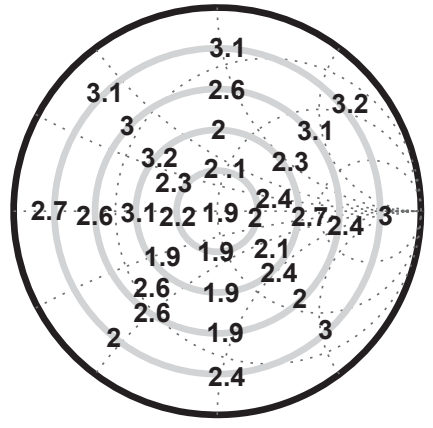

(a)

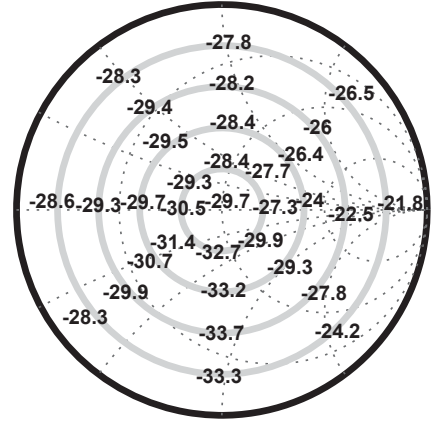

(b)
Fig. 6. Load-pull measurements for VSWR up to 9:1 without DPD with a 1 MHz 1024 QAM signal at 2 GHz. (a) EVM (\%), (b) ACPR (dB).

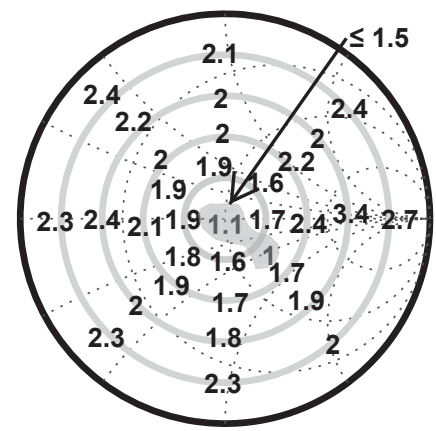

(a)

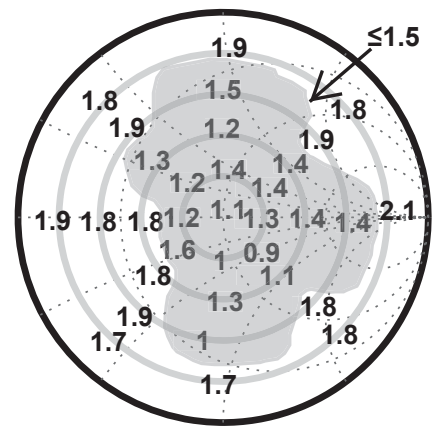

(b)
Fig. 7. Load-pull measurements of EVM (\%) for VSWR up to 9:1 with a 1 MHz 1024 QAM signal at $2 \mathrm{GHz}$. (a) after $50 \Omega$ optimized static DPD, (b) after ADPD.

DPD to $50 \Omega$ optimized static DPD to ADPD. Fig. 8 (a) and (b) show Smith charts of ACPR (dB) up to VSWR 9:1 with $50 \Omega$ optimized static DPD and with ADPD, respectively. For ACPR calculation, the higher and lower adjacent channel bandwidth is considered to be $1 \mathrm{MHz}$ each. The centers of adjacent channels are situated at an offset of $\pm 1.35 \mathrm{MHz}$ from the carrier frequency. The ACPR Smith charts show the area covered for values $\leq-34 \mathrm{~dB}$ and $\leq-35 \mathrm{~dB}$ for all three cases: without DPD, with $50 \Omega$ optimized static DPD and with ADPD. Similar to the Smith chart showing EVM, there is a significant increase in area under the shaded region for $\mathrm{ACPR} \leq-34 \mathrm{~dB}$ and $\mathrm{ACPR} \leq-35 \mathrm{~dB}$ from no DPD to $50 \Omega$ optimized static DPD to ADPD.

\section{CONCLUSION}

The linearity of high-efficiency class-E PAs can be drastically compromised in the presence of load mismatch. We presented a single-ended polar class-E PA with an on-chip integrated waveform characterizer, in $22 \mathrm{~nm}$ FDSOI CMOS technology to implement ADPD. For VSWR up to 9:1 when the PA is excited with a $1 \mathrm{MHz} 1024$ QAM RF signal at 2 $\mathrm{GHz}$, we showed that using our ADPD system sufficiently low EVM and ACPR values can be maintained across a relatively large area on the Smith chart compared to that when using a static (50 $\Omega$ optimized) DPD. The ADPD system can be used to optimize performance for relatively slow processes such as antenna load variation, temperature variation, and slow

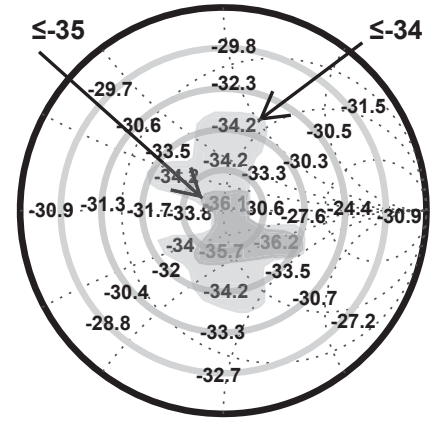

(a)

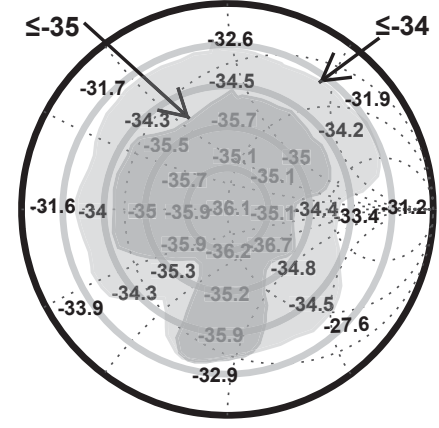

(b)
Fig. 8. Load-pull measurements of ACPR (dB) for VSWR up to 9:1 with a $1 \mathrm{MHz} 1024 \mathrm{QAM}$ signal at $2 \mathrm{GHz}$. (a) after $50 \Omega$ optimized static DPD, (b) after ADPD.

memory effects. This system allows practical application in next-generation mobile communication systems with complex modulation schemes that demand stringent linearity and are subjected to inevitable load mismatch.

\section{ACKNOWLEDGMENT}

We thank Global Foundries for Silicon donation, Gerard Wienk for CAD assistance, and Arnoud Rop for measurement support. This work is part of the research programme SHERPAS (12903) which is financed by the Dutch Research Council (NWO).

\section{REFERENCES}

[1] B. Razavi, RF microelectronics. Prentice Hall, 1998, vol. 2.

[2] P. B. Kenington, High linearity RF amplifier design. Artech House, 2000.

[3] S. C. Cripps, Advanced techniques in RF power amplifier design. Artech House, 2002

[4] K. R. Boyle et al., "Analysis of mobile phone antenna impedance variations with user proximity," IEEE Trans. Antennas Propag., vol. 55, no. 2, pp. 364-372, Feb 2007.

[5] A. Ghahremani et al., "Load mismatch sensitivity of class-E power amplifiers," IEEE Trans. Microw. Theory Techn., vol. 67, no. 1, pp. 216-230, Jan 2019.

[6] E. Zenteno et al., "Output impedance mismatch effects on the linearity performance of digitally predistorted power amplifiers," IEEE Trans. Microw. Theory Techn., vol. 63, no. 2, pp. 754-765, Feb 2015.

[7] C. Sánchez-Perez et al., "Improving digital predistortion mismatch sensitivity using tunable matching networks," 2011 IEEE 73rd Vehicular Technology Conference, pp. 1-5, 2011.

[8] A. Keerti and A. Pham, "Dynamic output phase to adaptively improve the linearity of power amplifier under antenna mismatch," in IEEE Radio Frequency Integrated Circuits Symp. - Dig. of Papers, June 2005, pp. 675-678.

[9] A. van Bezooijen et al., "Adaptively preserving power amplifier linearity under antenna mismatch," in 2004 IEEE MTT-S International Microwave Symposium Digest, vol. 3, June 2004, pp. 1515-1518.

[10] P. Reynaert and M. Steyaert, $R F$ power amplifiers for mobile communications. Springer Science \& Business Media, 2006.

[11] M. Huiskamp et al., "A delay spread cancelling waveform characterizer for RF power amplifiers," IEEE Trans. Circuits Syst. II, vol. 65, no. 12, pp. 1834-1838, Dec 2018 .

[12] E. L. Firrao et al., "An automatic antenna tuning system using only RF signal amplitudes," IEEE Trans. Circuits Syst. II, vol. 55, no. 9, pp. 833-837, Sep. 2008.

[13] F. Roger, "An analog approach to power amplifier predistortion," Microwave Journal, vol. 54, no. 4, pp. 60-76, 2011.

[14] J. Ponte et al., "Augmentation of class-E PA reliability under load mismatch conditions," in IEEE Int. Conf. on Electronics, Circuits and Systems, Dec 2018, pp. 33-36. 